\title{
Questionário de Hábitos de Estudo para estudantes universitários: validação e precisão ${ }^{1}$
}

\author{
André Luiz Moraes Ramos \\ Anelise de Barros Leite Nogueira ${ }^{2}$ \\ Denise Pereira de Alcântara Ferraz \\ Lígia Maria Teixeira de Faria Brezolin \\ Luciana Maria Pinto \\ Wilson de Freitas Muniz \\ UNISAL, Lorena-SP, Brasil
}

\begin{abstract}
Resumo: Este artigo apresenta e discute a construção de um instrumento para avaliação de hábitos de estudo. O questionário informatizado com o título Questionário de Hábitos de Estudo para estudantes universitários, composto por 97 itens em escala de tipo Likert, foi aplicado a 781 graduandos de 10 cursos de uma instituição particular do interior paulista, para avaliar os parâmetros psicométricos de precisão (consistência interna) e validade (análise fatorial). Os resultados apontaram nove fatores específicos com eigenvalue superior a 1,5. A consistência interna dos fatores apresentou alfa de Cronbach entre 0,603 e 0,934. Concluiu-se que o questionário apresenta parâmetros psicométricos satisfatórios, habilitando-o como instrumento útil na investigação de hábitos de estudo em universitários com as características da presente amostra.
\end{abstract}

Palavras-chave: hábitos de estudo, questionário, psicometria, validade do teste.

\section{Study Habits Questionnaire for college students: validation and reliability}

\begin{abstract}
This study presents and discusses the construction of an instrument to evaluate study habits. The digitalized questionnaire entitled Study Habits Questionnaire directed to college students, composed of 97 items with a Likert scale, was applied to 781 undergraduate students enrolled in 10 courses from a private college in the interior of São Paulo, Brazil to evaluate the reliability of psychometric parameters (internal consistency) and validity (factorial analysis). The results indicated nine specific factors with eigenvalue above 1.5. The internal consistency of the factors presented Cronbach's Alpha between 0.603 and 0.934 . The conclusion is that the questionnaire presents satisfactory psychometric parameters and is a useful instrument to investigate study habits in undergraduate students with characteristics similar to this study's sample.
\end{abstract}

Keywords: study habits, questionnaire, psychometrics, test validity.

\section{Cuestionario de Hábitos de Estudio para estudiantes universitarios: validad e precisión}

\begin{abstract}
Resumen: El objetivo del trabajo es presentar informaciones sobre la construcción de un instrumento para la evaluación de hábitos de estudio. El cuestionario informatizado titulado Cuestionario de Hábitos de Estudio para estudiantes universitarios, compuesto por 97 artículos en escala del tipo Likert, fue aplicado a 781 estudiantes universitarios de 10 cursos de una institución privada en São Paulo para evaluar los parámetros psicométricos de precisión (consistencia interna) y validad (análisis factorial). Los resultados indicaron 9 factores específicos con valores propios (eigenvalues) superiores a 1,5. La consistencia interna de los factores indicó alfa de Cronbach entre 0,603 y 0,934 . Se concluyó que el cuestionario presenta parámetros psicométricos satisfactorios, siendo así un instrumento de utilidad en la investigación de los hábitos de estudio de los estudiantes universitarios con las características de esta muestra.
\end{abstract}

Palabras clave: hábitos de estudio, cuestionario, psicometría, validación de test.

O ensino superior "abriu as portas", de modo especial, nas últimas décadas, abrangendo um maior número de

\footnotetext{
${ }^{1}$ Este texto foi revisado seguindo o Acordo Ortográfico da Língua Portuguesa (1990), em vigor a partir de $1^{\circ}$ de janeiro de 2009. Este artigo é resultado de projeto de pesquisa acerca dos hábitos de estudo dos universitários, desenvolvido entre 2006 e 2008, por todos os membros do Grupo de Pesquisa Dama (Desempenho Acadêmico e Metodologias Aplicadas), tendo sido apresentado no II Congresso Brasileiro de Psicologia: Ciência e Profissão em 2006. Os autores agradecem a colaboração de: Ariane Junqueira de Castro, Celso Eduardo Tuna, Elisabeth Hoffmann Sanches, Estefânia Maria de Almeida Gonçalves, Marcilene Rodrigues Pereira Bueno, Maria Aparecida Félix do Amaral e Silva, Maryah Bittencourt e Ana Valéria Sampaio de Almeida Reis.

${ }^{2}$ Endereço para correspondência

Profa. Anelise de Barros Leite Nogueira. Rua São Benedito, 326/apto 52. CEP 12.600-010. Lorena-SP, Brasil. E-mail: anelisedebarros@yahoo.com.br
}

pessoas de diferentes níveis sociais. Assim, a democratização deu mostras no ensino superior e a demanda foi se ampliando paulatinamente, afirmam Almeida e Soares (2004), sugerindo a ocorrência deste fato nos países em geral.

Esses autores, membros do Instituto de Educação e Psicologia da Universidade do Minho em Portugal, discutem o sucesso escolar e o desenvolvimento psicossocial dos universitários e destacam que

passamos de um "ensino de elite" para um "ensino de massas" (...); podemos reconhecer que não só alguns grupos de alunos se identificam pouco com a universidade, como esta ainda não se adaptou suficientemente no seu funcionamento para atender a esta nova realidade (Almeida \& Soares, 2004, p. 15). 
Apontam para a democratização do acesso, muito mais que para a democratização do sucesso na educação superior e destacam que os índices de fracasso escolar e de abandono sobressaem nos estratos sociais mais desfavorecidos. Completam, a partir de outros estudos (Braga da Cruz e cols., 1995; Direcção-Geral do Ensino Superior, 1999; Eurydice, 2000) salientando que, entre 1975 e 1997, Portugal aumentou geometricamente o número de universitários, chegando a 300.000 no final da década de noventa. Questionam, então, a massificação do ensino, em função não propriamente do número de alunos, mas, sim, do espaço físico, currículos e metodologias em iguais condições de quando se atendia apenas a elite social. O aluno da educação superior depara-se com exigências notadamente diferentes no nível social, pessoal e acadêmico. A transição do ensino médio para a educação superior é marcada pela confrontação com novas formas de avaliação, métodos de ensino diferentes daqueles a que o aluno estava acostumado, o que exige modificação de sua rotina e de seus hábitos de estudo.

Nessa linha de reflexão, Almeida e Soares (2004) ressaltam a capacitação socioprofissional dos estudantes e o próprio desenvolvimento dos jovens nos aspectos psíquico e social que, por sua vez, não se reduzem às aulas e atividades meramente curriculares. Reconhecem, entretanto, as dificuldades existentes por parte dos Sistemas de Ensino Superior, nos diversos países, em direção ao desenvolvimento psicossocial.

Santos (2000) afirma que atualmente atenta-se para os currículos dos cursos, processo ensino-aprendizagem e formas de avaliar, demandas sociais, atualização dos professores e envolvimento acadêmico dos estudantes, ressaltando que, após um crescimento em termos da quantidade, as universidades se orientam para a qualidade. Esta última observação, entretanto, mereceria ser investigada no caso da realidade brasileira, a começar pelos dados do Instituto de Estudos e Pesquisas Educacionais Anísio Teixeira (INEP) (Ministério da Educação, 2006, 2009) que se encontram adiante.

A respeito do envolvimento do estudante, Almeida (2002) comenta que as teorias atuais da aprendizagem salientam o papel ativo do aluno, o que implica a capacitação dele para assumir responsabilidades. Isto significa desenvolver comportamentos de maior autonomia chegando-se a uma aprendizagem autorregulada. Assim sendo, quanto mais o aluno conhece suas características, mais clareza terá em relação às tarefas escolares.

Visto que a educação superior não deve ter como meta unicamente a formação profissional, mas, também assegurar a aprendizagem de forma autônoma, é oportuno reportar ao estudo de Polydoro e Primi (2004), realizado em instituição privada de ensino superior do interior do estado de São Paulo, no período de 1998 a 1999, cujo objetivo era caracterizar o perfil psicológico e o nível de integração do estudante à universidade. Dentre várias considerações, os autores concluem que a organização, a persistência e a independência estão relacionadas à maior dedicação de tempo aos estudos e, ainda, admitem que os estudantes enfrentam várias mudanças, tais como " afastar-se de grupos de referência, conciliar trabalho, estudo e vida pessoal, estabelecer condições para o estudo (organização, local e tempo), superar déficit de habilidades básicas, enfim, responder às exigências de organização, gestão do tempo, autonomia e envolvimento" (p. 61).

Apesar de vários estudos recentes (Martins, Arroteia, \& Gonçalves, 1999; Monteiro, Vasconcelos, \& Almeida, 2005; Nogueira, 1998, 2002; Oliveira \& Oliveira, 2007; Vendramini e cols., 2004), de modo geral, a "população" de estudantes da educação superior ainda é pouco estudada (Polydoro \& Primi, 2004), apontando-se especialmente para as pesquisas brasileiras em comparação às investigações feitas com crianças e adolescentes, como salienta Nogueira (2002).

Há de se admitir que o conhecimento acerca do "perfil" do universitário, seja ele da universidade privada ou pública, passa a ser cada vez mais necessário, tendo-se em vista os objetivos de caráter profissional e institucional.

Nota-se o aumento progressivo deste público nas últimas décadas, especialmente no que tange à realidade brasileira, como informa o INEP (Ministério da Educação, 2006, 2009).

No Brasil, as instituições de ensino privadas mostraram um crescimento confirmado pelos índices estatísticos, compreendendo o período de 1997 a 2003, fornecidos pelo INEP (2006), que aponta para o total de 1.652 instituições nesta categoria. Dentre estas, 1.302 são particulares e 350, comunitárias, confessionais e filantrópicas. Observa-se que o mesmo Instituto apresenta o total de 207 instituições públicas, entre federais, estaduais e municipais, no mesmo período.

Em 2009, o INEP disponibilizou um relatório técnico no qual aponta para um levantamento de dados da educação superior de 2002 a 2008. Neste constam 2.252 instituições de ensino superior (IES), sendo 236 públicas (federais, estaduais e municipais) e 2016 privadas (particulares, comunitárias, confessionais e filantrópicas), salientando-se o total de 1.579 no caso das particulares. Ainda, neste relatório há menção à evolução do número de concluintes (entre 2002 e 2008), passando de 466.260 (2002) para 800.318 (em 2008) e na categoria "privada" de 315.159 (2002) para 612.560 (2008).

O relatório alerta para uma diminuição de 29 instituições em relação a 2007, mostrando uma desaceleração das IES até 2007, e ressalta que no ano de 2008, pela primeira vez desde 1997, este número diminuiu, sobretudo em relação às federais, em função de integração de instituições, fusão, ou compra, notadas nos últimos anos, especialmente com a criação de Institutos Federais de Educação Tecnológica. Entretanto, observou-se uma ampliação na região Nordeste, com 10 instituições a mais do que em 2007.

Vale destacar a seguinte observação presente neste relatório: "o declínio no número das IES (...) não se refletiu na oferta de vagas, número de ingressos, matrículas e concluintes, que mantiveram um crescimento semelhante aos dos anos anteriores" (Ministério da Educação, 2009).

O estudante universitário faz-se conhecer pelas suas características pessoais, incluindo-se neste rol suas motivações, preferências, necessidades diversas, conjunto de valores, condições para agir no contexto acadêmico, limitações, 
dentre outros fatores. Desta forma, vários "recortes" podem ser feitos quando se pensa em analisar um dos polos do processo ensino-aprendizagem: o estudante ou aprendiz.

Em um estudo acerca dos caminhos da universidade para o século XXI, sob a visão de educadores brasileiros, os autores afirmam que "há uma série de problemas que se constituem em desafios para a educação superior, que necessita rever sua missão para atender à sociedade do século XXI, sociedade do conhecimento, da informação e, principalmente, da educação" (Lima, Castro, \& Carvalho, 2000, p. 14).

A relação desse protagonista com os professores, com os pares, com a instituição, com o seu processo formativo, com a futura profissão passa necessariamente pela autopercepção e autoavaliação. Nessa linha da percepção de si, há, por exemplo, pesquisas sobre autoconceito e autoestima (Campbell \& Lavallee, 1993; Cerqueira, 1996; Crano, Crano, \& Biaggio, 1983; Ingraham, 1988; La Rosa, \& Díaz-Loving, 1991; Nogueira, 2002; Ramos, 1997).

Ao lado dessas questões, Vendramini e cols. (2004) avaliam a vida acadêmica considerando dez aspectos e, dentre eles, a habilidade para estudo (percepção pessoal de competências cognitivas) e condições de estudo (disponibilidade de tempo e investimento em atividades de aprendizagem), tendo como objetivo a construção e validação de uma Escala de Avaliação da Vida Acadêmica (EAVA). Nesse sentido é pertinente a compreensão das modalidades, estilos, e ações rotineiras, priorizadas ao estudar.

Muitas instituições, nas mais variadas discussões acerca do ensino-aprendizagem, descrevem a necessidade de uma avaliação efetiva que contribua com a caracterização da sua população, assim como com a possibilidade de implementação de programas de orientação quanto aos hábitos de estudo. No entanto, mesmo em face de tais necessidades, muitas são as dificuldades de se investigar o fenômeno, devido à escassez de instrumentos para avaliação de hábitos de estudo. Deste modo, a questão que norteou este estudo foi a seguinte: o Questionário de Hábitos de Estudo para estudantes universitários, em formato Likert, é válido e apresenta precisão?

Ao se delimitar o problema proposto, registra-se o direcionamento do estudo à amostra composta por estudantes brasileiros, de instituição particular de ensino superior, tomando-se como objeto a validade de construto e a precisão, com análise da consistência interna, aferida pelo cálculo do coeficiente alfa de Cronbach $(\alpha)$. Desta forma, o objetivo deste trabalho é apresentar dados sobre a construção de um instrumento para avaliação de hábitos de estudo. O instrumento é descrito na próxima seção, incluindo-se dados históricos de sua construção.

\section{Método}

\section{Amostra}

A pesquisa foi realizada com 781 alunos de uma instituição particular do interior paulista, na qual predominam cursos das áreas Humanas e Exatas, sendo que o curso de Psicologia pode ser identificado como pertencente à área de Saúde. A distribuição dos sujeitos é apresentada na Tabela 1 (a amostra foi composta em função da disponibilidade dos alunos e dos coordenadores em participarem da pesquisa, conforme distribuição).

Tabela 1

Composição da amostra

\begin{tabular}{lccc}
\hline \multicolumn{2}{c}{ Característica } & \multirow{c}{c}{ Frequência } & Porcentagem \\
\hline $\begin{array}{l}\text { Licenciatura } \\
\text { em Ciências }\end{array}$ & Cursos & & \\
Humanas & $\begin{array}{c}\text { Geografia } \\
\text { História }\end{array}$ & & $34,3 \%$ \\
& $\begin{array}{l}\text { Pedagogia } \\
\text { Ciências da Saúde }\end{array}$ & & \\
Ciências Exatas & Psicologia & 212 & $27,1 \%$ \\
& $\begin{array}{c}\text { Matemática } \\
\text { Computação }\end{array}$ & 201 & $25,7 \%$ \\
Ciências Sociais e & Turismo & 100 & $12,8 \%$ \\
Aplicadas & Administração & & \\
& Direito & & \\
\hline
\end{tabular}

Ao se considerar a especificidade da amostra, de acordo com a Tabela 1 , nota-se que predominam alunos $(34,3 \%)$ do curso de Licenciatura em Ciências Humanas (Filosofia, Geografia, História e Pedagogia), seguido pelo curso de Psicologia, (27,1\%), de Ciências Exatas $(25,7 \%)$ e da área de Ciências Sociais e Aplicadas, que engloba os cursos de Turismo, Administração e Direito (12,8\%).

Entre os sujeitos pesquisados, a maioria é do sexo feminino $(58,8 \%)$, tem idade variando de 17 a 55 anos, com média de 24 anos e 11 meses (desvio padrão de 6 anos e 11 meses), estuda no período noturno (72,2\%), sendo $27,2 \%$ de calouros, $47,9 \%$ das séries intermediárias e $24,9 \%$ de formandos. Com relação à realização de atividade profissional, apesar de a maioria não trabalhar (57,5\%), a maior parte da amostra realiza atividades domésticas $(69,9 \%)$ e colabora com o sustento familiar $(59,5 \%)$.

$\mathrm{O}$ número de componentes da amostra atendeu às especificações de Dancey e Reidy (2006), que prevê um mínimo de 100 participantes na análise e ter pelo menos cinco vezes mais participantes do que variáveis. Tomando como base que o instrumento tem 97 itens, a amostra ultrapassou em muito o número mínimo de 485 sujeitos pesquisados.

\section{Instrumento}

Para a avaliação de hábitos de estudos foi elaborado o Questionário de Hábitos de Estudo para estudantes universitários. O instrumento piloto era composto por 97 itens em escala com cinco alternativas de resposta do tipo Likert 
(sempre, frequentemente, algumas vezes, poucas vezes, nunca ou quase nunca).

O referido instrumento, elaborado pelos autores deste artigo que, por sua vez, contaram com as contribuições de Tierno (2003), em especial, quanto ao primeiro conjunto de itens, inicialmente foi constituído de 12 fatores os quais seguem na seguinte ordem: interesse geral para aprender; organização e planejamento de estudo; uso de tecnologias; atenção e esforço em classe; facilidade para memorizar e recordar o aprendido; leitura com compreensão eficaz; estudo individual em casa; preparação para as avaliações; busca pela atualização acadêmico-profissional; anotações da matéria e leituras; flexibilidade e iniciativa ao estudar; estudo em grupo; todos atinentes ao tema "hábitos de estudo", organizados em "janelas" identificadas por letras alfabéticas em que somente o seu completo preenchimento permitia prosseguir para o próximo item e conjunto de questões pertinentes.

Salienta-se que a versão final do instrumento conta com 9 fatores e 56 itens, valendo ressaltar o processo de construção desse material, com revisões sistemáticas por parte do grupo autor do estudo, quanto ao aspecto semântico dos itens, sintaxe, número de itens e organização destes em "tópicos ou categorias", tendo em vista a avaliação dos hábitos de estudo da amostra eleita.

Em retrospectiva, destacam-se as contribuições de Nogueira (1998, 2002) e Sampaio (2003) com estudos direcionados, de modo especial, ao estudante universitário para a fase inicial ou primeira etapa da construção do instrumento, objeto do presente trabalho. No ano de 2004, esses autores optaram por adaptar um questionário intitulado "Para se conhecer melhor como estudante", da autoria de Tierno (2003). $\mathrm{O}$ autor apresenta esse instrumento quando faz referência à "metacognição" na obra que traz como título: As melhores técnicas de estudo: Saber ler corretamente, fazer anotações e preparar-se para os exames, publicada no Brasil pela editora Martins Fontes. A primeira edição é espanhola, com o título Las mejores técnicas de estudio, e data de 1994.

$\mathrm{O}$ instrumento "Para se conhecer melhor como estudante" conta com 64 questões, distribuídas em conjuntos ou tópicos (total - 8), sendo que em cada tópico constam oito questões. Os títulos dos tópicos são: "Interesse geral por aprender", "Organização e planejamento do estudo", "Atenção e esforço em classe", "Facilidade para memorizar e recordar o aprendido", "Leitura com compreensão eficaz", "Estudo individual em casa", "Controles e exames", "Autoconfiança e autoestima".

Após analisarem a estrutura do instrumento citado, Nogueira e Sampaio (2004) mantiveram o Sistema Likert 5 pontos propostos por Tierno (2003). No entanto, decidiram ampliar o número de tópicos, assim como alteraram o aspecto substantivo de parte deles, com revisões e modificações nas questões, e, ainda, atribuição de novas nomenclaturas.

A nova versão, adaptada por Nogueira e Sampaio (2004), foi constituída de 96 questões (itens), distribuídas em 12 tópicos. Manteve-se o conjunto de oito questões para cada tópico. E assim, o instrumento recebeu o título Hábitos de Estudo em Universitários e passou a ser identificado como uma Escala Likert. Essa versão passou a ser analisada pelo grupo de pesquisa em 2006, quando assumiu a tarefa de dar continuidade às revisões sobre a estrutura do instrumento, como já mencionado anteriormente.

\section{Procedimento}

\section{Coleta de dados}

Inicialmente, os pesquisadores foram às salas de aula e informaram aos alunos a finalidade da pesquisa e os convidaram a participar da aplicação do instrumento. Os horários de aplicação da pesquisa foram previamente combinados entre os pesquisadores, as coordenações de cada curso, e os responsáveis pelos laboratórios de informática. No caso dos cursos com poucos participantes, eles foram reunidos para uma única sessão de aplicação.

Em um dia de aula dos alunos, os autores do questionário realizaram a aplicação coletiva do instrumento nos laboratórios de informática da referida instituição de ensino superior. Houve durante a aplicação o cuidado para captar as respostas que melhor refletissem seus hábitos de estudo. Como instrução de aplicação, os universitários foram orientados a preencher o questionário, optando pela alternativa que melhor representasse sua postura atual frente aos estudos.

\section{Análise dos dados}

Para o tratamento dos dados desta pesquisa, foi utilizado o programa Statistical Package for Social Science (SPSS), versão 11.0 para o Windows. Em uma reunião da qual participaram os pesquisadores, os coordenadores de curso e a direção acadêmica da instituição, foram divulgados os resultados da pesquisa. Os coordenadores de curso foram orientados por estes autores quanto ao fenômeno avaliado, relatividade das respostas, contingências durante a coleta, e cautela na avaliação dos hábitos de estudo. Ademais, destacou-se a perspectiva de outras aplicações nos próximos semestres e turmas para comparações, análises e acompanhamento dessa dimensão do desempenho acadêmico (análises por parte do colegiado de seus cursos e procedimentos similares).

\section{Considerações éticas}

Salienta-se que o projeto relativo ao presente estudo foi aprovado pelo Comitê de Ética em Pesquisa da UNITAU-SP ( $\mathrm{n}^{\mathrm{o}}$ protocolo 385/06 de 06/11/06). Após a explicação dos procedimentos da pesquisa, foi garantida aos participantes a confidencialidade dos resultados e a liberdade de decisão em participar da pesquisa. Todos os alunos que foram ao laboratório aceitaram participar da pesquisa e assinaram o Termo de Consentimento Livre e Esclarecido, conforme recomenda 
a Resolução 16 de dezembro de 2000, publicada em 2001 (Conselho Federal de Psicologia, Caderno Especial de Resoluções, 2001), atrelada à Resolução do Conselho Nacional de Saúde (CNS/196).

\section{Resultados e Discussão}

\section{Validade do Questionário de Hábitos de Estudo}

A fim de determinar se o método de análise fatorial é adequado para tratar os dados obtidos no Questionário de Hábitos de Estudo, foi, inicialmente, realizada uma análise fatorial por meio do método dos componentes principais (PC), procedendo-se ao cálculo de Kaiser-Mayer-Olkin (KMO) e ao Teste de Esfericidade de Bartlett. O KMO trabalha com as correlações parciais das variáveis e são considerados aceitos índices iguais ou superiores a 0,60 ; enquanto que o teste de Bartelett testa a hipótese de que a matriz de covariâncias é uma matriz de identidade (Tabachnick \& Fidell, 1996).

Os resultados apontaram um valor de 0,900 no teste de $\mathrm{KMO}$ e o teste de esfericidade permitiu rejeitar a hipótese $\left(\chi_{(4656)}^{2}=27492,676, p<0,001\right)$, indicando que a matriz de correlações não é a matriz de identidade. Com base nesses dados, pôde-se, então, prosseguir a análise fatorial, que demonstrou estar adequada aos dados obtidos via aplicação do instrumento. A identificação dos fatores que compõem o questionário foi feita por meio da análise fatorial dos eixos principais (PAF), com rotação oblíqua (OBLIMIN), por se considerar que os fatores podem estar correlacionados. Foram retidos 11 fatores com eigenvalue superior a 1,5 , conforme descrito na Tabela 2.

Tabela 2

Composição fatorial do Questionário de Hábitos de Estudo

\begin{tabular}{cccccc}
\hline Fator & Número de itens & Eigenvalue & $\begin{array}{c}\text { \% Variância total } \\
\text { explicada }\end{array}$ & Variância acumulada & Coeficiente alfa $(\alpha)$ \\
\hline 1 & 11 & 14,754 & 15,210 & 15,210 & 0,852 \\
2 & 11 & 5,667 & 5,842 & 21,052 & 0,842 \\
3 & 4 & 4,349 & 4,484 & 25,536 & 0,711 \\
4 & 5 & 3,071 & 3,166 & 28,702 & 0,777 \\
5 & 5 & 2,898 & 2,988 & 31,690 & $0,732^{*}$ \\
6 & 5 & 2,502 & 2,579 & 34,269 & 0,714 \\
7 & 5 & 2,197 & 2,265 & 36,534 & 0,606 \\
1 & 4 & 1,930 & 1,989 & 38,523 & 0,660 \\
11 & 5 & 1,722 & 1,775 & 40,298 & $0,51,992$ \\
\end{tabular}

* Fatores com consistência interna insatisfatória.

Esses 11 fatores, inicialmente, explicam 43,476\% do construto hábitos de estudos. Observa-se que apenas os quatro primeiros fatores explicam, cada um, $3 \%$ da variância total do fator, enquanto os outros sete fatores não possuem o mesmo nível de explicação, devendo os resultados por estes fornecidos serem considerados com cautela.

\section{Precisão do Questionário de Hábitos de Estudo}

Os fatores foram analisados, ainda, quanto à sua consistência interna, aferida pelo cálculo do coeficiente alfa de Cronbach ( $\alpha$ ). Cronbach (1996) e Churchil (1999) consideram aceitáveis valores entre 0,60 e 0,80 .

Assim sendo, o fator $5(\alpha=0,482)$ e o fator $10(\alpha=0,524)$ não apresentaram coeficientes de precisão satisfatórios, sendo desconsiderados nas análises subsequentes. Nota-se, todavia, que a exclusão dos fatores 5 e 10 reduz o poder explicativo do questionário para $38,864 \%$ do fenômeno investigado, valor que ainda representa um elevado poder explicativo do instrumento, quase $40 \%$ do construto hábitos de estudo.

Foi, também, avaliada a correlação entre o escore dos sujeitos em cada item do fator e o seu escore total no fator, cujos valores devem ficar acima de 0,30 (correlação itemtotal). Ademais, investigou-se o quanto a precisão do instrumento seria afetada, caso o item fosse excluído, de modo que o item só seria mantido no fator se sua exclusão não representasse um ganho significativo na consistência interna dele (alfa se item retirado), tornando a consistência significativamente superior a 0,30 no fator analisado.

\section{Composição fatorial do Questionário de Hábitos de Estudo}

Em sua versão final, o instrumento é composto por nove fatores com cargas fatoriais, eigenvalue, superiores a 1,5 e precisão satisfatória (alfa superior a $0,60 \mathrm{em}$ todos os fatores), o que se observa na Tabela 3. 
Tabela 3

Versão final do Questionário de Hábitos de Estudo

\begin{tabular}{lccc}
\hline \multicolumn{1}{c}{ Fator } & $\begin{array}{c}\text { Número } \\
\text { de itens }\end{array}$ & Média & Desvio-padrão \\
\hline 1. Planejamento do estudo & 11 & 2,84 & 0,73 \\
2. Dificuldades no uso de & 11 & 2,58 & 0,59 \\
técnicas e estratégias de estudo & & 2,72 & 0,78 \\
3. Uso de tecnologias & 4 & 3,94 & 0,69 \\
4. Estudo em grupo & 5 & 3,61 & 0,78 \\
5. Condições ambientais & 5 & & \\
adequadas para o estudo & & 3,55 & 0,86 \\
$\begin{array}{l}\text { 6. Falta de atualização do } \\
\text { conhecimento }\end{array}$ & 5 & & \\
$\begin{array}{l}\text { 7. Dificuldade de organização } \\
\text { espaço-temporal }\end{array}$ & 4 & 3,08 & 0,76 \\
8. Desatenção e desmotivação & 5 & 2,07 & 0,53 \\
9. Anotação da matéria & 6 & 4,12 & 0,79 \\
\hline
\end{tabular}

A nomeação dos fatores, segundo Dancey e Reidy (2006), deve ser realizada a partir da verificação do que os itens relacionados têm em comum. A seguir são apresentados os nove fatores e a sua interpretação semântica:

Fator 1: planejamento do estudo

O primeiro fator é composto por onze itens que enfocam o planejamento do estudo e apresentam uma organização prévia, um plano de ação, incluindo também o uso de roteiros e revisões periódicas dos conteúdos estudados, conforme atestam os itens com maior carga fatorial: Faço um roteiro personalizado de cada tema estudado (45); Elaboro meu plano de estudo para cada matéria (9); Realizo autoavaliações mensais ou semestrais para rever o meu grau de aproveitamento nos estudos (15); Repasso, periodicamente, cada tema (14).

Fator 2: dificuldades no uso de técnicas e estratégias de estudo

Os onze itens que compõem o fator 2 reportam-se às dificuldades no uso de técnicas e estratégias de estudo enfrentadas pelos sujeitos investigados, em termos de leitura, avaliações, aplicação do conhecimento estudado, entre outras. Os itens mais fortes nesse fator são: Dificilmente faço resumo das leituras que realizo (78); Depois da primeira leitura de um texto, encontro dificuldades para ter uma idéia geral sobre o seu conteúdo (40); Dificilmente faço esquemas das leituras que realizo (79); Memorizo os conteúdos acadêmicos com dificuldade (35).

\section{Fator 3: uso de tecnologias}

Semanticamente, os quatro itens que compõem o terceiro fator retratam o uso de tecnologias, manifestado por meio dos recursos que a informática oferece à área da educação, como importante parceiro para a construção do conhecimento. São itens desse fator: Visito sites específicos da minha área (21); Tenho aproveitamento acadêmico quando navego na internet (22); Frequento cursos a distância, via internet (23); Discuto a matéria abordada em sala, trocando e-mails ou participando de chats com os colegas (18).

\section{Fator 4: estudo em grupo}

O quarto fator, constituído de cinco itens, retrata o estudo em grupo, apontando que o trabalho colaborativo na companhia de colegas permite acréscimo à aprendizagem. Os itens mais representativos desse fator são: A reflexão feita em grupo favorece para mim e meus colegas a fixação do conteúdo (92); Quando estudo em grupo para as avaliações, contribuo com os conhecimentos que tenho da matéria (91); Colaboro para que cada componente do grupo desenvolva habilidades de expor idéias, criticá-las, e tirar conclusões (94); O meu estudo individual tem facilitado o meu estudo em grupo (96).

\section{Fator 5: condições ambientais adequadas para o estudo}

O quinto fator, identificado como condições ambientais adequadas para o estudo, engloba questões relativas ao horário e espaço físico onde o estudo se realiza. Dentre os cinco itens desse fator encontram-se: Ao estudar, cerco-me de silêncio (51); Ao estudar, evito, ao máximo, distrações (53); A temperatura do ambiente em que estudo é agradável (52); Tenho um lugar específico para estudar (49).

\section{Fator 6: falta de atualização do conhecimento}

Esse fator retrata a ausência de atualização dos conteúdos do curso por meio da falta de busca de complementação aos assuntos abordados em aula, denominado falta de atualização do conhecimento, manifestada na baixa frequência com que apresenta os comportamentos apontados em quatro dos cinco itens: Leio ou assino revistas específicas de minha área de formação (89); Faço assinatura de pelo menos uma Revista Científica relacionada ao Curso de Graduação que frequento (71); Frequento, no período de dois anos, pelo menos um Congresso relativo à minha profissão (72); Procuro assistir a simpósios, palestras, oficinas, congressos, cursos de extensão ou similares com vistas a ampliar meus conhecimentos ou participar deles (90).

\section{Fator 7: dificuldade de organização espaço-temporal}

Os quatro itens que compõem esse fator, a saber: Meus horários destinados aos estudos e revisão da matéria são irregulares e indeterminados (11); Meu tempo de estudo é menor do que eu realmente necessito (4); O estudo individual das matérias está atrasado (59); O local de estudo é desorganizado (50), referem-se à organização e cumprimento de um 
cronograma de estudos adequado às necessidades do universitário, sendo que essa falta de organização estende-se ao próprio local de estudo. Por isso, esse fator foi identificado como dificuldade de organização espaço-temporal.

\section{Fator 8: desatenção e desmotivação}

Foram observadas nesse fator a desatenção e desmotivação apresentadas pelos alunos para conduzir os estudos. Isso pôde ser verificado por meio da baixa frequência com que os alunos manifestaram ao responderem aos comportamentos apresentados em três dos cinco itens do referido fator: Presto atenção às explicações durante a aula (24); Gosto de aprender sobre tudo (1); Presto atenção àquilo que o professor prioriza (29).

\section{Fator 9: anotação da matéria}

O nono e último fator da escala reporta-se à participação ativa do aluno em sala de aula por meio do registro dos conteúdos abordados, recebendo, por isso, o nome de anotar a matéria. Os seis itens com maior carga nesse fator foram: Anoto o conteúdo apresentado pelo professor em sala de aula (76); Anoto em meu caderno os conteúdos desenvolvidos em sala de aula (19); Estudo a partir das anotações que fiz (77); Volto às anotações feitas por mim, revejo-as e utilizoas (30).

A literatura nessa área traz importantes contribuições, verificando-se preocupações semelhantes ao presente estudo, no que tange ao uso de instrumentos que contemplam aspectos dos métodos de estudo e as possíveis relações com o rendimento acadêmico.

Monteiro, Vasconcelos e Almeida (2005), ao discutirem a influência dos métodos de estudo no rendimento acadêmico, salientam a dificuldade de se interferir nas variáveis relativas à trajetória ou percurso pessoal e admitem as possibilidades de identificação e intervenção dos fatores de natureza acadêmica que, por sua vez, igualmente interferem na formação. Nessa direção, fizeram uso do Inventário de Atitudes e Comportamentos Habituais de Estudo (IACHE) da autoria de Tavares, Almeida, Vasconcelos e Bessa (2004), também com adoção do Sistema Likert 5 pontos, concluindo que, no âmbito universitário, a forma como a aprendizagem é abordada constitui-se em importante fator para o rendimento acadêmico e variabilidade das classificações escolares. A esse respeito, destacam que os alunos que apresentaram abordagem mais superficial ao estudo, com maior pontuação no enfoque reprodutivo, revelaram um padrão geral de rendimento acadêmico inferior.

Vendramini e cols. (2004) apresentam um estudo feito com o objetivo de construir e analisar as propriedades psicométricas de um instrumento de medida acerca da percepção do estudante sobre sua experiência universitária. Esse instrumento foi intitulado Escala de Avaliação da Vida Acadêmica (EAVA). Dentre os fatores obtidos, constaram "envolvimento em atividades não obrigatórias" (extracurriculares ou complementares) e "condições para o estudo e desempenho acadêmico". Os autores destacam os resultados satisfatórios, com identificação de um índice de consistência interna de 0,87 , com variação nos cinco fatores entre 0,63 e 0,80 . Alertam, entretanto, para o quinto fator - "condições para o estudo e desempenho acadêmico" - com apresentação da menor consistência interna $(0,63)$, e sugerem a inclusão de novos itens em função de esse fator ser composto de apenas quatro itens.

No presente estudo, os fatores com a mesma natureza do quinto fator apresentado por Vendramini e cols. (2004) são os seguintes: "condições ambientais adequadas para o estudo" (Fator 5), com $\alpha$ 0,482 (consistência interna insatisfatória) e "dificuldade de organização espaço-temporal" (Fator 7), com $\alpha$ 0,714, ambos com cinco itens, conforme mostra a Tabela 2.

Outro fator, no estudo citado, que mostra alguma correspondência ou similaridade em relação a presente pesquisa, é aquele denominado "envolvimento em atividades não obrigatórias" (extracurriculares ou complementares), com cinco itens e $\alpha 0,71$. Neste trabalho a identificação é referente ao fator 6 "falta de atualização do conhecimento", que conta com cinco itens também, sendo o $\alpha 0,730$.

No presente estudo, o fator 3 "uso de tecnologias", com quatro itens e $\alpha$ 0,711, mostra-se necessário e, ao mesmo tempo, uma novidade na construção desta modalidade de instrumento, levando-se em conta o uso de ferramentas virtuais por parte dos universitários em sua rotina de trabalhos acadêmicos na atualidade.

O Conselho Federal de Psicologia tem-se empenhado na regulamentação dos instrumentos utilizados para a avaliação psicológica e publicou a Resolução CFP 02/2003 que regulamenta, entre outras questões, a elaboração de instrumentos psicológicos. Desde então, percebe-se uma maior atenção em relação à avaliação. Este trabalho, portanto, insere-se nesta perspectiva de construção de instrumentos que obedecem ao processo de validação determinado pelo órgão máximo da psicologia no país.

A continuidade dos estudos e investigações com o público universitário nas diferentes áreas do saber será fundamental para esclarecimentos e ampliação dos conhecimentos acerca de suas características quanto aos hábitos e formas de estudar e aprender.

\section{Considerações finais}

Com os valores obtidos nas análises psicométricas realizadas, conclui-se que os dados obtidos no Questionário de Hábitos de Estudo estão adequados para a realização de uma análise dos componentes principais, de acordo com os cálculos de Kaiser-Mayer-Olkin (KMO) e com o Teste de Esfericidade de Bartelett. Por meio da análise fatorial dos eixos principais (PAF), com rotação oblíqua (OBLIMIN), foram inicialmente identificados 11 fatores: nove apresentaram 
carga fatorial satisfatória (eigenvalue superior a 1,5) e coeficientes alfa de Cronbach superiores a 0,60.

Com base nos resultados, pode-se concluir que o Questionário de Hábitos de Estudo é um instrumento que apresenta índices psicométricos que confirmam sua validade de construto. $\mathrm{O}$ instrumento, em sua versão final, consta de nove fatores consistentes, que demonstraram ter precisão satisfatória. Os dados acima expostos indicam que o questionário apresenta as propriedades psicométricas adequadas para uma medida psicológica.

Portanto, esse instrumento está capacitado para avaliar o fenômeno de hábitos de estudos com as características da presente amostra quanto ao planejamento do estudo, dificuldades no uso de técnicas de estudo, uso de tecnologias, estudo em grupo, condições ambientais adequadas para o estudo, falta de atualização do conhecimento, dificuldade de organização espaço-temporal, desatenção e desmotivação, anotação da matéria.

\section{Referências}

Almeida, L. S. (2002). Facilitar a aprendizagem: Ajudar os alunos a aprender e a pensar. Psicologia Educacional e Escolar, 6(2), 155-165.

Almeida, L. S., \& Soares, A. P. (2004). Os estudantes universitários: Sucesso escolar e desenvolvimento psicossocial. In E. Mercuri \& S. A. J. Polydoro (Orgs.), Estudante universitário: Características e experiências de formação (pp. 15-40). Taubaté, SP: Cabral Editora e Livraria Universitária.

Braga da Cruz, M., Cruzeiro, M. E., Ramos, A., Leandro, E., Nunes, J. S., Matias, N., Pedroso, P., Robinson, M. G., \& Cavaco, V. (1995). O desenvolvimento do ensino superior em Portugal: Situação e problemas de acesso. Lisboa: Departamento de Programação e Gestão Financeira, Ministério da Educação.

Campbell, J. D., \& Lavallee, L. F. (1993). Who am I? The role of self-concept confusion in understanding the behavior of people with low self-esteem. In R. F. Baumeister (Ed.), Self-Esteem: The puzzle of low self-regard (pp. 3-20). New York: Plenum Press.

Cerqueira, T. C. S. (1996). Possíveis influências do autoconceito e do locus de controle sobre o rendimento acadêmico. In Anais do Congresso Nacional de Psicologia Escolar - Prevenção e Saúde Mental na Educação, 3, 89-90. Rio de Janeiro: UERJ.

Churchil, G. A., Jr. (1999). Marketing research: Methodological foundations (7th ed.). New York: InterThomson Publishing.

Conselho Federal de Psicologia. (2001). Caderno especial de resoluções (Resolução CFP No. 016/2000 atrelada à Resolução MS 196/96 do CNS). Jornal do Conselho Federal de Psicologia, 9-10.
Conselho de Federal de Psicologia. (2003). Resolução CFP No. 002/2003. Recuperado em 24 janeiro 2008, de http:// www.pol.org.br/pol/export/sites/default/pol/legislacao/ legislacaoDocumentos/resolucao2003_02.pdf

Crano, S. L., Crano, W. D., \& Biaggio, A. M. B. (1983). Desenvolvimento de uma medida de autoconceito em português. Educação e Realidade, 8(3), 33-44.

Cronbach, L. J. (1996). Fundamentos da testagem psicológica (5a ed., C. A. Silveira Neto \& M. A. V. Veronese, Trads.). Porto Alegre: Artes Médicas.

Dancey, C. P., \& Reidy, J. (2006). Estatística sem matemática para psicologia: Usando SPSS para Windows (3a ed., L. Viali, Trad.). Porto Alegre: Artmed.

Direcção-Geral do Ensino Superior (1999). O ensino superior em Portugal. Lisboa: Ministério da Educação, DirecçãoGeral do Ensino Superior.

Eurydice (2000). Key data on education in the European Union. European Commission.

Ingraham, C. L. (1988). Self-esteem, crisis and school performance. In J. Sandoval (Ed.), Handbook of crisis, counseling, intervention and prevention in the schools (pp. 21-30). London: LAE.

La Rosa, J., \& Díaz-Loving, R. (1991). Evaluacion del autoncepto: Una escala multidimensional. Revista Latinoamericana de Psicologia, 23(1), 15-33.

Lima, P. G., Castro, F., \& Carvalho, M. A. V. (2000). Caminhos da universidade rumo ao século XXI: Pontos e estratégias para sua orientação na visão de educadores brasileiros. Paidéia (Ribeirão Preto), 10(18), 8-27.

Martins, A. M., Arroteia, J. C., \& Gonçalves, M. M. B. (1999). Práticas socioculturais e escolares dos estudantes universitários: Estudo de caso (Coleção Universidade Hoje, Série Contributos). Aveiro, Portugal: Universidade de Aveiro.

Ministério da Educação. Instituto Nacional de Estudos e Pesquisas Educacionais Anísio Teixeira. (2006). Censo da educação superior 2006. Recuperado em 14 setembro 2006, de http:/www.inep.gov.br/imprensa/noticias/ censo/superior/news04_05.htm

Ministério da Educação. Instituto Nacional de Estudos e Pesquisas Educacionais Anísio Teixeira. (2009). Resumo técnico - Censo da educação superior 2008: Dados preliminares. Recuperado em 2 maio 2010, de http:// www.inep.gov.br/imprensa/noticias/censo/superior/ news04_05.htm

Monteiro, S., Vasconcelos, R. M., \& Almeida, L. S. (2005). Rendimento acadêmico: Influência dos métodos de estudos. Actas do Congresso Galaico Português de Psicopedagogia, 8, 14-16.

Nogueira, A. B. L. (1998). Criatividade e percepção em estudantes de Psicologia. Lorena, SP: Stiliano/Centro UNISAL.

Nogueira, A. B. L. (2002). Criatividade, autoestima e rendimento acadêmico: Um estudo com universitários de psicologia. Tese de doutorado não publicada, Instituto de Psicologia, Universidade de São Paulo, São Paulo. 
Nogueira, A. B. L., \& Sampaio, R. G. (2004). Hábitos de estudo em universitários - primeiro estudo piloto. Curso de Psicologia, LEP, UNISAL, Lorena, SP. (Material apostilado).

Oliveira, K. L., \& Oliveira, R. A. M. (2007). Propriedades psicométricas de uma escala de condições de estudo para universitários. Avaliação Psicológica, 6(2), 181-188.

Polydoro, S. A. J., \& Primi, R. (2004). Integração ao ensino superior: Explorando sua relação com características de personalidade e envolvimento acadêmico. In E. Mercuri \& S. A. J. Polydoro (Orgs.), Estudante universitário: Características e experiências de formação (pp. 41-66). Taubaté, SP: Cabral Editora e Livraria Universitária.

Ramos, A. L. M. (1997). Escala Janis-Field revisada por Eagly: Discriminação dos itens, precisão e validade. Revista da Faculdade Salesiana, 25(35), 67-78.

Sampaio, R. G. (2003). Como estudar. Taubaté, SP:Academia do Pensar/Cabral Editora e Livraria Universitária.

Santos, S. M. (2000). As responsabilidades da universidade no acesso ao ensino superior. In A. P. Soares, A. Osório, J. V. Capela, L. S. Almeida, R. M. Vasconcelos, \& S. M. Caíres (Orgs.), Transição para o ensino superior (pp. 1-17). Braga, Portugal: Universidade do Minho.

Tabachnick, B. G., \& Fidel, L. S. (1996). Using multivariate statistic. California: Allyn \& Bacon.

Tavares, J., Almeida, L.S., Vasconcelos, R. \& Bessa, J. (2004). Inventário de atitudes e comportamentos habituais de estudo - IACHE. Aveiro, Portugal: Universidade de Aveiro. Braga, Portugal: Universidade do Minho.

Tierno, B. (2003). As melhores técnicas de estudo: Saber ler corretamente, fazer anotações e preparar-se para os exames (C. Schilling, Trad.). São Paulo: Martins Fontes. Vendramini, C. M. M., Santos, A. A. A., Polydoro, S. A. J., Sbardelini, E. T. B., Serpa, M. N. F., \& Natário, E. G. (2004). Construção e validação de uma escala sobre avaliação da vida acadêmica - EAVA. Estudos de Psicologia, 9(2), 259-268.

André Luiz Moraes Ramos é Doutor em Psicologia pela Universidade de Brasília.

Anelise de Barros Leite Nogueira é Doutora em Psicologia Escolar e do Desenvolvimento Humano pela Universidade de São Paulo.

Denise Pereira de Alcântara Ferraz é doutoranda em Psicologia Social pela Universidade Estadual do Rio de Janeiro.

Lígia Maria Teixeira de Faria Brezolin é Mestre em Tecnologias da Inteligência e Design Digital pela Pontifícia Universidade Católica de São Paulo.

Luciana Maria Pinto é psicóloga pelo Centro Universitário Salesiano de São Paulo de Lorena.

Wilson de Freitas Muniz é Mestre em Ciências Espaciais pelo Instituto Nacional de Pesquisas Espaciais.
Recebido: 05/03/2010

$1^{a}$ revisão: $18 / 05 / 2010$

$2^{a}$ revisão: $13 / 02 / 2011$

Aceite final: 05/04/2011 